Jeronymo Marcondes Pinto

aMinistério do Trabalho, Secretaria de Inspeção do Trabalho, Superintendência Regional do Trabalho em São Paulo. São Paulo, SP, Brasil.

Contato:

Jeronymo Marcondes Pinto

E-mail:

jeronymomp@gmail.com

O autor declara que o trabalho não foi subsidiado e que não há conflitos de interesses.

O autor informa que o trabalho não foi apresentado em eventos científicos.

\section{Tendência na incidência de acidentes e doenças de trabalho no Brasil: aplicação do filtro Hodrick- -Prescott}

\author{
Trend in the incidence of accidents and work-related diseases in \\ Brazil: application of the Hodrick-Prescott filter
}

\section{Resumo}

Introdução: analisar a tendência temporal da incidência de acidentes do trabalho é importante para prognósticos de longo prazo. No entanto, alterações cíclicas e sazonais, como o trabalho informal e a subnotificação dos acidentes, podem influenciar e precisam ser consideradas. O método do filtro Hodrick-Prescott (HP), utilizado para avaliar a dinâmica de variáveis macroeconômicas, pode propiciar análise mais precisa da evolução de variáveis sujeitas a essas flutuações do que métodos usualmente utilizados em epidemiologia, como o de médias móveis (MM). Objetivo: testar o filtro HP como método de extração de tendência da variável "incidência de acidentes de trabalho". Métodos: análise comparativa entre o filtro HP e o método MM para extrair a tendência de longo prazo das variáveis acidente típico, de trajeto, doença do trabalho e total de acidentes no Brasil entre 2008 e 2013. Resultados: ambas metodologias indicaram tendências semelhantes das variáveis ao longo do período. No entanto, a análise da tendência de longo prazo pelo método MM pode ser mais afetada por flutuações de curto prazo. Conclusão: o método HP permite inferir de maneira mais consistente a tendência de médio e longo prazo da variável, o que pode torná-lo mais adequado para a definição e a gestão de políticas públicas.

Palavras-chave: acidente de trabalho; doença do trabalho; análise estatística; estudos de série temporal; computação matemática.

\begin{abstract}
Introduction: analyzing the temporal trend of occupational accidents incidence is important for long-term prognostics. However, cyclical and seasonal changes, such as informal work and the underreporting of accidents, can affect it and need to be considered. The Hodrick-Prescott (HP) filter method, used to assess the dynamics of macroeconomic variables, can provide a more precise analysis of the evolution of variables subject to these fluctuations than methods commonly used in epidemiology, such as that of moving averages (MA). Objective: to test the HP filter as trend extraction method for the variable "incidence of occupational accidents". Methods: comparative analysis between the HP filter and the MA method to extract the long-term trend of the following variables: typical accident, commuting accident, occupational disease, and total accidents in Brazil between 2008 and 2013. Results: both methodologies indicated similar trends of the variables over the period. However, the analysis of the long-term trend by the MA method may be more affected by short-term fluctuations. Conclusion: the HP method allows to more consistently infer the medium- and long-term trend of the variable "incidence of occupational accidents", which can make it more suitable for proposing and managing public policies.
\end{abstract}

Keywords: work accident; occupational disease; statistical analysis; time series studies; mathematical computing. 


\section{Introdução}

De acordo com a Organização Internacional do Trabalho (OIT) $)^{11}$, cerca de 2,3 milhões de pessoas morrem por ano em decorrência de acidentes de trabalho e 860 mil pessoas sofrem algum ferimento no trabalho todos os dias. Os custos dessas ocorrências são alarmantes, sendo estimados em 2,8 trilhões de dólares por ano. A OIT ${ }^{11}$ destaca o Brasil como um país que contribui significativamente para essa estatística, com mais de 700 mil acidentes e adoecimentos relacionados ao trabalho por ano - o país ocupa o quarto lugar do ranking mundial de acidentes de trabalho com óbito, ficando atrás apenas da China, Estados Unidos e Rússia.

Almeida e Barbosa-Branco ${ }^{2}$ estimaram que até 4\% do Produto Interno Bruto (PIB) dos países seja perdido devido a consequências econômicas do acidente de trabalho, podendo chegar a $10 \%$ em países em desenvolvimento.

Os mesmos autores ${ }^{2}$ também destacam que, apesar de o número de acidentes do trabalho ser elevado no mundo todo, o Brasil apresenta uma média de acidentes elevada com relação aos demais países. Segundo dados do Anuário Estatístico da Previdência Social (AEPS) ${ }^{3}$, em 2013, o Brasil registrou 737.378 acidentes de trabalho liquidados ${ }^{\mathrm{b}}$, incluindo 2.897 óbitos e 14.837 geradores de incapacidade permanente.

Dada a magnitude dos números relativos a acidentes de trabalho no país, muitos estudos se dedicaram a avaliar a tendência temporal dessa variável. A ideia básica seria averiguar a dinâmica das variáveis relativas a acidentes de trabalho no Brasil nos últimos anos e realizar prognósticos de possíveis comportamentos futuros. Um levantamento bibliográfico baseado nessa linha de pesquisa foi feito por Santana e colegas ${ }^{4}$. Estes autores inferiram que, apesar de algumas estatísticas de acidentes de trabalho indicarem tendência decrescente desses acidentes no Brasil, podem não apontar o real comportamento da variável. Muitos fatores podem influenciar o resultado, como a informalidade, a subnotificação de acidentes de trabalho, dentre outros ${ }^{4}$.

Pereira ${ }^{5}$ analisou a evolução dos acidentes entre 1997 e 2008. Segundo o autor, a mortalidade e a taxa de letalidade diminuíram entre 2002 e 2008; no entanto, o número absoluto de acidentes de trabalho liquidados teve aumento acentuado no período devido sobretudo à mudança na forma de classificar os benefícios acidentários pela Previdência.
Por outro lado, ao avaliar a tendência da variável "número de acidentes de trabalho", Wünsch Filho ${ }^{6}$ contrapôs a visão tradicional de que somente fatores ligados ao ambiente e às relações de trabalho influenciariam, abordando a variável de forma ampla, estatisticamente relacionada a variáveis macroeconômicas e macrossociais, como o PIB. A dinâmica descrita baseou-se na constatação de que, em períodos de crescimento econômico, há aumento da população ocupada, ao contrário dos períodos de recessão, quando ocorre o inverso. Quando aumenta a formalização do mercado de trabalho e, por conseguinte, a população exposta a riscos ocupacionais, aumentam também os acidentes desse tipo, bem como a quantidade efetivamente notificada.

Guimarães e colaboradores ${ }^{7}$ propõem três metodologias diferentes para analisar a tendência de um indicador relativo à mortalidade: método de médias móveis (MM), regressão polinomial e joinpoint regression. Esses autores se basearam na análise de tendência da incidência de mortalidade advinda de câncer de colo de útero. Entretanto, o estudo serviu de base para outros autores no que se refere às metodologias para avaliação da tendência de acidentes de trabalho no Brasil. Como exemplo, pode-se citar Almeida e colaboradores ${ }^{8}$, que usaram o método de joinpoint regression para avaliar a tendência de acidentes de trabalho no Brasil, discriminando a análise em acidentes típicos, de trajeto, totais e com óbito. Como conclusão, indicam que todos os tipos de acidente foram reduzidos, à exceção de acidentes de trajeto, no período entre 1998 e 2008.

Contudo, não é de conhecimento deste autor nenhum estudo que tenha realizado a análise de tendência do número de acidentes de trabalho no Brasil por meio do filtro Hodrick-Prescott (HP) ${ }^{9}$. Esse método de "filtragem" é muito utilizado para avaliar a dinâmica de variáveis macroeconômicas e consiste em extrair a tendência estocástica de uma série temporal, que terá variações suaves ao longo do tempo, mas que não serão correlacionadas com suas variações cíclicas. Ao realizar esse processo, é possível analisar o componente de tendência de uma série temporal expurgado dos efeitos cíclicos naturais de qualquer variável avaliada ao longo do tempo, o que permite uma inferência mais precisa da evolução da quantidade de acidentes de trabalho no Brasil.

Siqueira ${ }^{10}$ faz uma análise semelhante, comparando o filtro HP com método de MM para análise de dados climáticos, considerando que, tal como no caso da Epidemiologia, o filtro HP não é comumente utilizado na climatologia. $\mathrm{O}$ aprofundamento no estudo de métodos que permitam analisar a

b Acidentes de trabalho liquidados correspondem ao número de acidentes cujos processos foram encerrados administrativamente pelo INSS, depois de completado o tratamento e indenizadas as sequelas. 
tendência dos dados relativos a acidentes de trabalho no Brasil é essencial para planejar políticas públicas em saúde e segurança no trabalho. O filtro HP e o método de MM têm como vantagem a operacionalização automática com o uso de sistemas informatizados, o que não é o caso de regressões lineares e não lineares, por exemplo. Isso que facilita a criação de softwares que possam fazer esse cálculo estatístico independentemente da atuação humana.

\section{Acidentes de trabalho no Brasil}

Esses dados ressaltam a necessidade de um sistema de controle de estatísticas e indicadores consistentes para avaliar a eficiência e a eficácia das políticas públicas aplicadas ao setor.

Segundo Pereira ${ }^{5}$, a Previdência Social vem desempenhando, nos últimos quarenta anos, papel importante no que se refere ao armazenamento e geração de dados e estatísticas sobre saúde e segurança no trabalho no Brasil. Nesse sentido, a lei estabeleceu a obrigatoriedade de as empresas informarem à Previdência Social todos os acidentes de trabalho ocorridos. Em caso de acidente de trabalho, a empresa deve preencher a Comunicação de Acidente de Trabalho (CAT), de forma a suprir a Previdência com as informações necessárias para conceder benefícios, bem como gerar estatísticas importantes.

Com o apoio da Empresa de Tecnologia e Informações da Previdência Social (Dataprev), o INSS iniciou um processo de modernização na forma de comunicar acidentes, culminando no atual Catweb, sistema baseado em um aplicativo que permite a geração eletrônica da CAT e o seu registro via internet.

Os dados referentes à quantificação de acidentes de trabalho no Brasil têm sido sumarizados em publicações da Previdência Social, como o AEPS $^{3}$ e o Anuário Estatístico de Acidentes de Trabalho $(\mathrm{AEAT})^{12}$, por exemplo. Esses dados têm sido usados em pesquisas científicas na área de saúde e segurança no trabalho ${ }^{13}$.

Segundo Pereira ${ }^{5}$, uma limitação importante relacionada ao banco de dados da CAT como base de estudos de acidentes de trabalho no Brasil é sua limitada abrangência. Cabe destacar que a CAT não é preenchida para trabalhadores que não estão vinculados ao Regime Geral de Previdência Social (RGPS). Assim, essas estatísticas não levam em conta os militares e servidores públicos, nem os trabalhadores vinculados ao RGPS mas que não têm cobertura contra acidentes, os chamados "outros contribuintes”, como autônomos, empresários e contribuintes facultativos.
Destaca-se ainda a impossibilidade desse sistema conter informações relativas às ocorrências de acidentes de trabalho no setor informal. Em 2016, de acordo com a Pesquisa Mensal de Emprego do Instituto Brasileiro de Geografia e Estatística (IBGE) $^{14}$, a taxa de informalidade do Brasil era de aproximadamente 19\%, esta parte da população ocupada estava fora das estatísticas oficiais de acidentes. Algumas pesquisas visam avaliar o impacto da precarização das relações de trabalho, como a informalidade, sobre a incidência de acidentes de trabalho, porém de forma predominantemente descritiva, dada a ausência de dados oficiais ${ }^{15,16}$.

Também é preciso considerar que além das já citadas limitações do banco de dados da CAT, o fato da notificação partir do empregador gera subnotificação. Segundo Neto et al. ${ }^{17}$, a lei 8.213/1991 estabeleceu que a emissão da CAT era condição necessária para conceder benefícios previdenciários e garantir estabilidade no emprego. Esse contexto pode desfavorecer a notificação dos acidentes pelas empresas, a fim de evitar impactos financeiros adicionais.

A introdução do Nexo Técnico Epidemiológico (NTEP), com a lei $11.430 / 2006^{18}$, teve o intuito de diminuir o impacto da subnotificação de acidentes de trabalho no Brasil. Segundo Todeschini, Lino e Melo ${ }^{19}$, a ideia básica do NTEP centra-se na hipótese de que toda vez que fosse identificada uma incidência elevada de determinada doença no Sistema Único de Benefícios da Previdência Social haveria o enquadramento dessa doença como de natureza acidentária. Portanto, o NTEP relaciona determinada doença às atividades em que ocorre com maior incidência, por meio do cruzamento do diagnóstico médico, com descrição na Classificação Internacional de Doenças (CID), com sua incidência estatística dentro da Classificação Nacional de Atividades Econômicas $(\mathrm{CNAE})^{17}$. Assim, o perito do Instituto Nacional do Seguro Social (INSS) pode, com base nas prerrogativas legais atinentes a seu cargo, considerar determinado acidente ou doença como advindo do trabalho, independentemente da emissão da CAT. Com isso o NTEP garantiria mais credibilidade aos bancos de dados oficiais da Previdência relativos a acidentes do trabalho.

Entretanto, mesmo com a introdução do NTEP, ressalta-se que as informações obtidas por meio desse banco de dados possuem limitações, dadas as exclusões citadas no início desta seção (servidores públicos, trabalhadores informais, entre outros). Além disso, por ser a metodologia do NTEP unicamente baseada em estatísticas, não garante que todos os casos de acidentes de trabalho não comunicados serão computados, mas somente aqueles mais comuns em determinada atividade econômica (CNAE). Assim, qualquer avaliação estatística com 
base nos dados do AEPS deve ter suas conclusões restritas somente à sua abrangência.

Nesse contexto, este estudo teve por objetivo extrair a tendência de variáveis relativas a acidentes típicos, de trajeto e de doenças do trabalho no Brasil, entre 2008 e 2013, por meio do filtro HodrickPrescott (HP), método não usual na análise epidemiológica, e comparar o resultado com o método de médias móveis (MM), já amplamente empregado para este fim, com o propósito de verificar a aplicabilidade do filtro HP para essa finalidade.

\section{Métodos}

O presente trabalho tem caráter de análise metodológica.

\section{Análises}

A ideia que embasa a filtragem da tendência de uma série de tempo $\left(Y_{t}\right)$ é a de que qualquer série temporal pode ser decomposta em dois componentes:

$$
Y_{t}=f\left(C_{t}, \tau_{t}\right)
$$

sendo $C_{t}$ o componente cíclico e a tendência.

Uma das formas de se obter o componente é pelo método de médias móveis (MM).

O principal objetivo do método MM simples é fornecer o valor médio da variável observada em determinado período de tempo ${ }^{7}$. Com efeito, para cada valor mais recente incluído no cálculo da média, um valor mais antigo é excluído, sendo que todas as observações têm o mesmo peso. Esse método permite eliminar grandes flutuações nos valores da variável, extraindo somente o movimento principal.

Essa decomposição utilizada pressupõe um processo aditivo nos componentes da série de tempo, podendo ser especificado da seguinte forma:

$$
Y_{t}=C_{t}+\tau_{t}
$$

Apontada por alguns autores ${ }^{7}$, a desvantagem desse método MM é que só demonstra uma tendência de curto prazo se não houver muitas observações, o que inviabiliza a sua aplicação para avaliação de longo prazo da variável em estudo, cuja quantidade de observações não seja muito numerosa, mas seja significativa. Essa característica é muito comum na maior parte das metodologias tradicionalmente usadas para avaliar a tendência de acidentes de trabalho, o que pode afetar parte dos resultados atingidos, pois os bancos de dados dessa variável no Brasil tendem a ser muito limitados.

Alternativamente, a tendência pode ser tratada como uma função aleatória do tempo, o que implicaria alterações suaves em seu valor, mas não correlacionadas com o ciclo. Um exemplo seria o passeio aleatório:

$$
Y_{t}=Y_{t-1}+e_{t}
$$

Na equação acima, a tendência da série varia de forma estocástica com o passar do tempo, não sendo indicada como variação constante.

Para se extrair a tendência de uma série dessa natureza, pode-se utilizar o filtro HP, ou seja, um algoritmo que minimiza a seguinte relação a $\tau$ :

$$
\min _{T}\left\{\sum_{t=1}^{T}\left(Y_{t}-\tau_{t}\right)^{2}+\lambda \sum_{t=2}^{T-1}\left[\left(\tau_{t+1}-\tau_{t}\right)-\left(\tau_{t}-\tau_{t-1}\right)\right]^{2}\right\}
$$

Ou também denotado como:

$$
\min _{T}\left\{\sum_{t=1}^{T}\left(Y_{t}-\tau_{t}\right)^{2}+\lambda \sum_{t=2}^{T-1}\left(\tau_{t+1}-2 \tau_{t}+\tau_{t-1}\right)-\left(\tau_{t}-\tau_{t-1}\right)^{2}\right\}
$$

Os valores de tendência computados por esse método serão aqueles que minimizam os valores dessa função para cada ponto. Assim, esse método irá gerar uma sequência de $\tau_{t}$ que minimiza a soma de quadrados da referida expressão. O parâmetro $\lambda$ é um fator de "custo" ou "penalidade" em computar flutuações na tendência. Basicamente, quando $\lambda \rightarrow 0$, a soma dos quadrados é minimizada quando $Y_{t}=\tau_{t}$, ou seja, a tendência coincide com a própria série. Por outro lado, quando $\lambda \rightarrow \infty$, a tendência é totalmente independente do ciclo, tornando-se constante.

A avaliação de tendência de uma série temporal não pode prescindir de uma análise de estacionariedade da série. Segundo Enders ${ }^{20}$, uma série estacionária é aquela que possui função média constante e finita e função autocovariância dependente apenas do valor de defasagem. Analiticamente, uma série $\left(Y_{t}\right)$ será dita estacionária se e somente se:

$$
\left\{\begin{array}{c}
E\left(y_{t}\right)=\mu=\text { constante } \\
\operatorname{Cov}\left(y_{t}, y_{t+\tau}\right)=f(\tau)=\text { função da defasagem }
\end{array}\right\}
$$

Basicamente, essa característica implica que choques externos sobre a variável em análise não 
serão perpetuados indefinidamente no valor final da variável. Com efeito, a avaliação de tendência de uma variável pode ser afetada por choques externos, sendo necessário verificar a sua estacionariedade. O teste de Dickey-Fuller Aumentado (Augmented Dickey-Fuller [ADF]) testa a hipótese nula de que a série não é estacionária.

Os cálculos para a análise proposta foram realizados por meio do programa $R^{21}$. O método MM foi aplicado à série de dados por meio do pacote "stats", presente no programa $R$. No caso do filtro HP, usou-se o "mFilter", também presente no programa $R$.

Portanto, a aplicação dos métodos de MM e do filtro HP permite a decomposição da série temporal, extraindo a componente descrita. O período usado para calcular a MM foi determinado automaticamente por meio de um algoritmo do pacote "stats", bem como o valor ótimo de, determinado por um algoritmo do pacote "mFilter".

\section{Dados utilizados}

Foram utilizadas as quantidades mensais de acidentes de trabalho registradas entre janeiro de 2008 e dezembro de 2013, classificando a análise por acidentes típicos, acidentes de trajeto e doenças do trabalho. Esses dados estão disponíveis no AEPS e foram extraídos do site do Ministério da Previdência Social ${ }^{3}$. A escolha desse período para a análise deriva da necessidade de não influenciar os resultados com efeitos oriundos da aplicação do Nexo Técnico Epidemiológico (NTEP) pelo Ministério da Previdência Social a partir de 2007, o que afetou o número de acidentes notificados de forma muito expressiva. Qualquer avaliação na tendência do número de acidentes de trabalho comunicados à Previdência Social deve levar em conta o efeito de quebra estrutural ocasionado por tal evento.

De acordo com a lei 8.213/1991' , a empresa deve comunicar acidentes de trabalho ocorridos à Previdência Social até o primeiro dia útil seguinte à ocorrência. Segundo o AEPS ${ }^{3}$, esses acidentes podem ser classificados de três formas diferentes:

- Acidentes típicos: decorrem da atividade profissional desempenhada pelo acidentado.

- Acidentes de trajeto: ocorrem no trajeto entre a residência e o local de trabalho do segurado e vice-versa.

- Acidentes devidos à doença do trabalho: ocasionados por qualquer tipo de doença profissional peculiar a determinado ramo de atividade constante na tabela da Previdência Social.

Além disso, foi avaliada uma série que consistiu no somatório dos acidentes típicos, de trajeto e das doenças do trabalho com os acidentes configurados como acidentes de trabalho pelo NTEP - os chamados "acidentes sem CAT".

- Acidentes totais: somatório de acidentes típicos, de trajeto, de doenças do trabalho e dos acidentes sem CAT.

Neste estudo foi utilizada uma variável relativa à incidência de acidentes de trabalho. Essa variável foi calculada com base no quociente entre os números de acidentes de trabalho para uma determinada competência e a quantidade de contribuintes empregados inscritos na Previdência Social em determinado ano. A variável calculada baseou-se na seguinte fórmula:

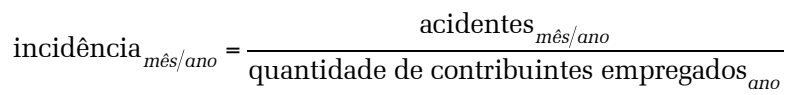

Com o intuito de facilitar a visualização dos dados, esse indicador foi multiplicado por um fator de 100.000, dado que o denominador é muito maior do que o numerador.

A fórmula desse indicador baseia-se no método descrito em Chagas, Servo e Salim ${ }^{22}$ para "Indicadores da saúde". Segundo esses autores, no numerador devem constar todas as ocorrências registradas em determinado acidente, enquanto que no denominador está computada a quantidade de contribuintes cobertos contra acidentes de trabalho, sendo excluídos os servidores públicos estatutários, contribuintes individuais e militares.

\section{Resultados}

\section{Análise descritiva}

A evolução das ocorrências de acidentes de trabalho no Brasil pode ser observada por meio do comportamento dos acidentes típicos, de trajeto, das doenças ocupacionais e dos acidentes totais dados, respectivamente, pelas variáveis "Incidência de acidentes típicos" (Figura 1), "Incidência de acidentes de trajeto" (Figura 2), "Incidência de doenças ocupacionais" (Figura 3) e "Incidência de acidentes totais" (Figura 4). As estatísticas descritivas para as séries em questão encontram-se na Tabela 1. 


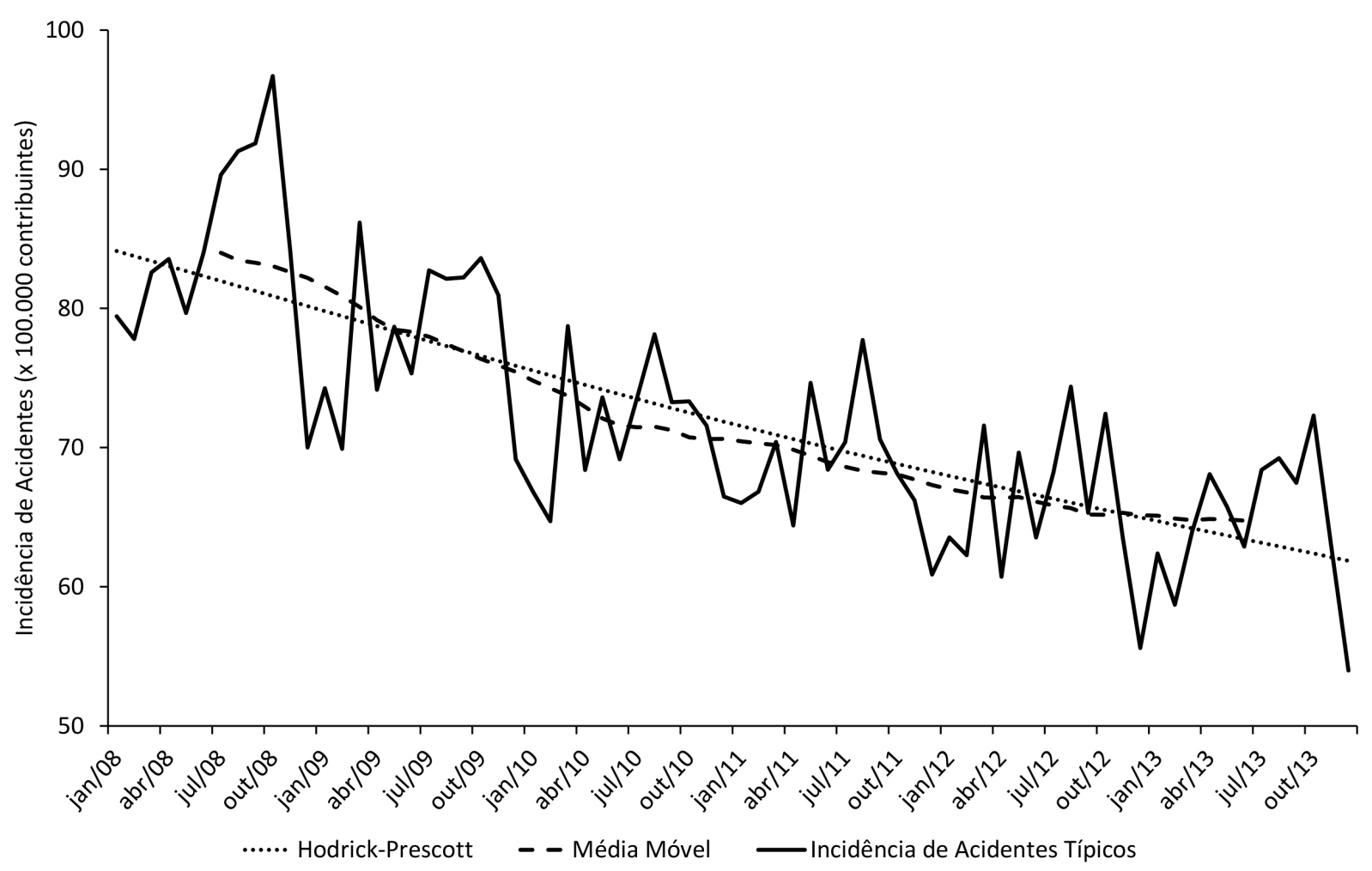

Figura 1 Nível e tendência da incidência de acidentes de trabalho típicos no Brasil de janeiro de 2008 a dezembro de 2013

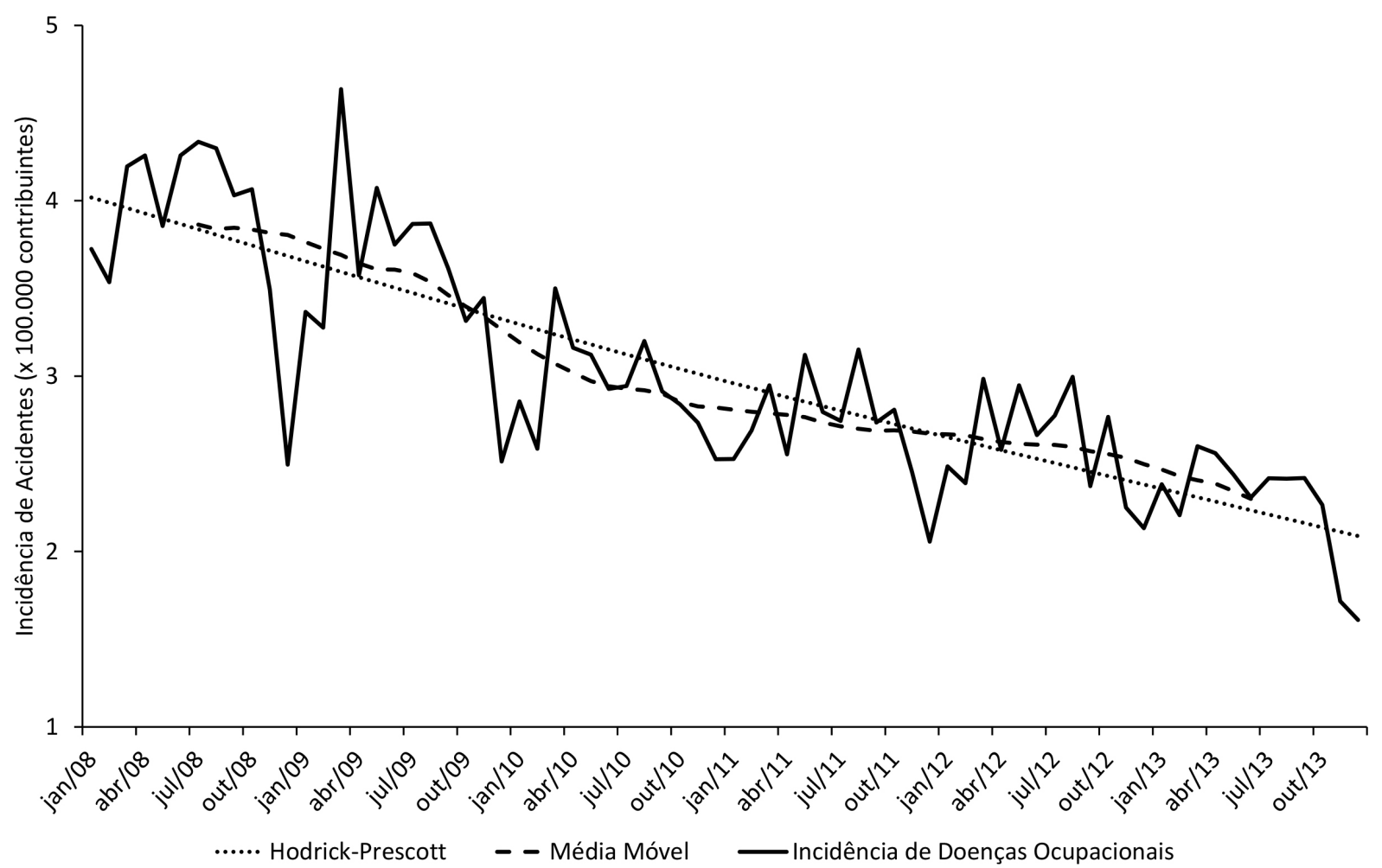

Figura 2 Nível e tendência da incidência de doenças ocupacionais no Brasil de janeiro de 2008 a dezembro de 2013 


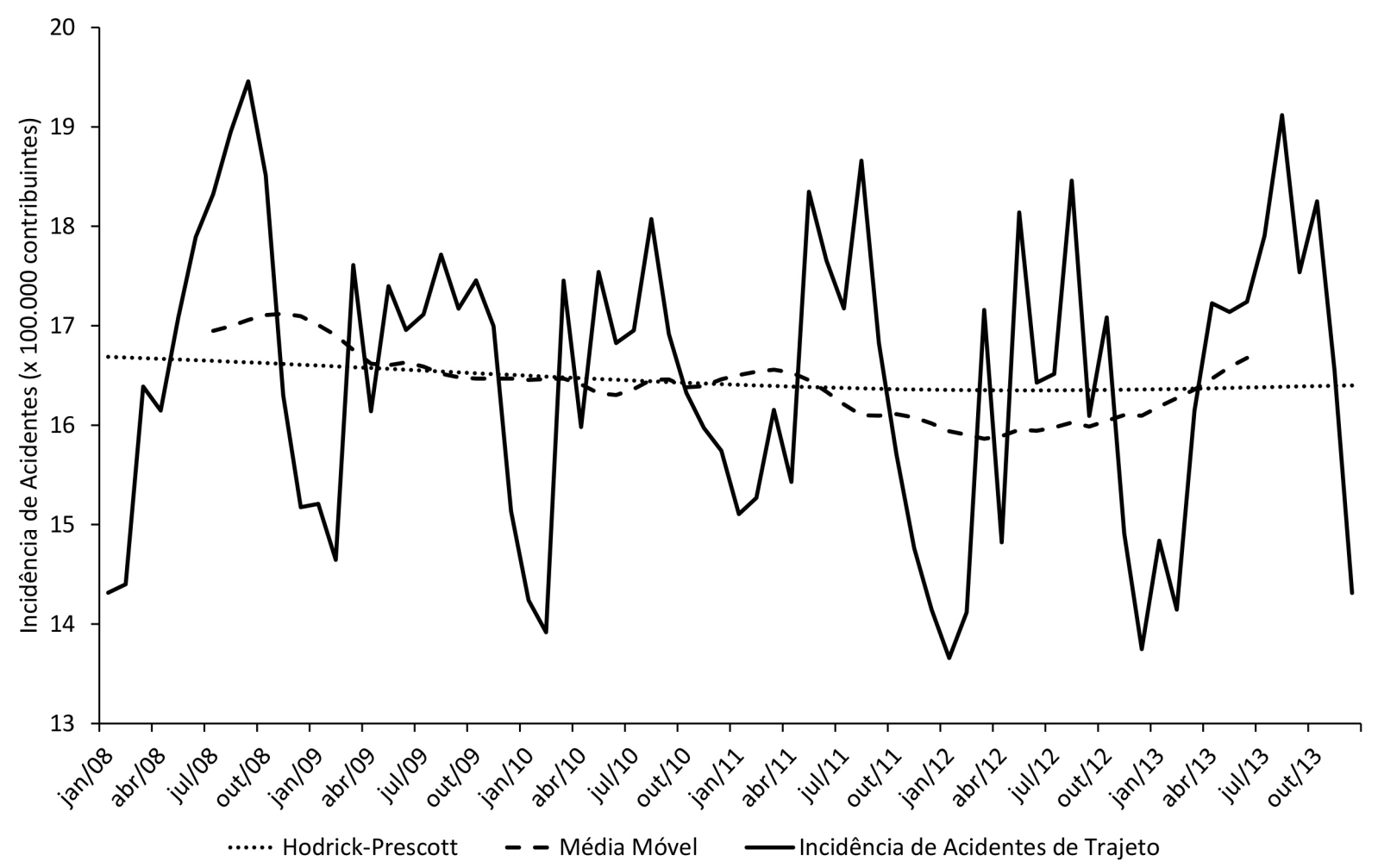

Figura 3 Nível e tendência da incidência de acidentes de trajeto no Brasil de janeiro de 2008 a dezembro de 2013

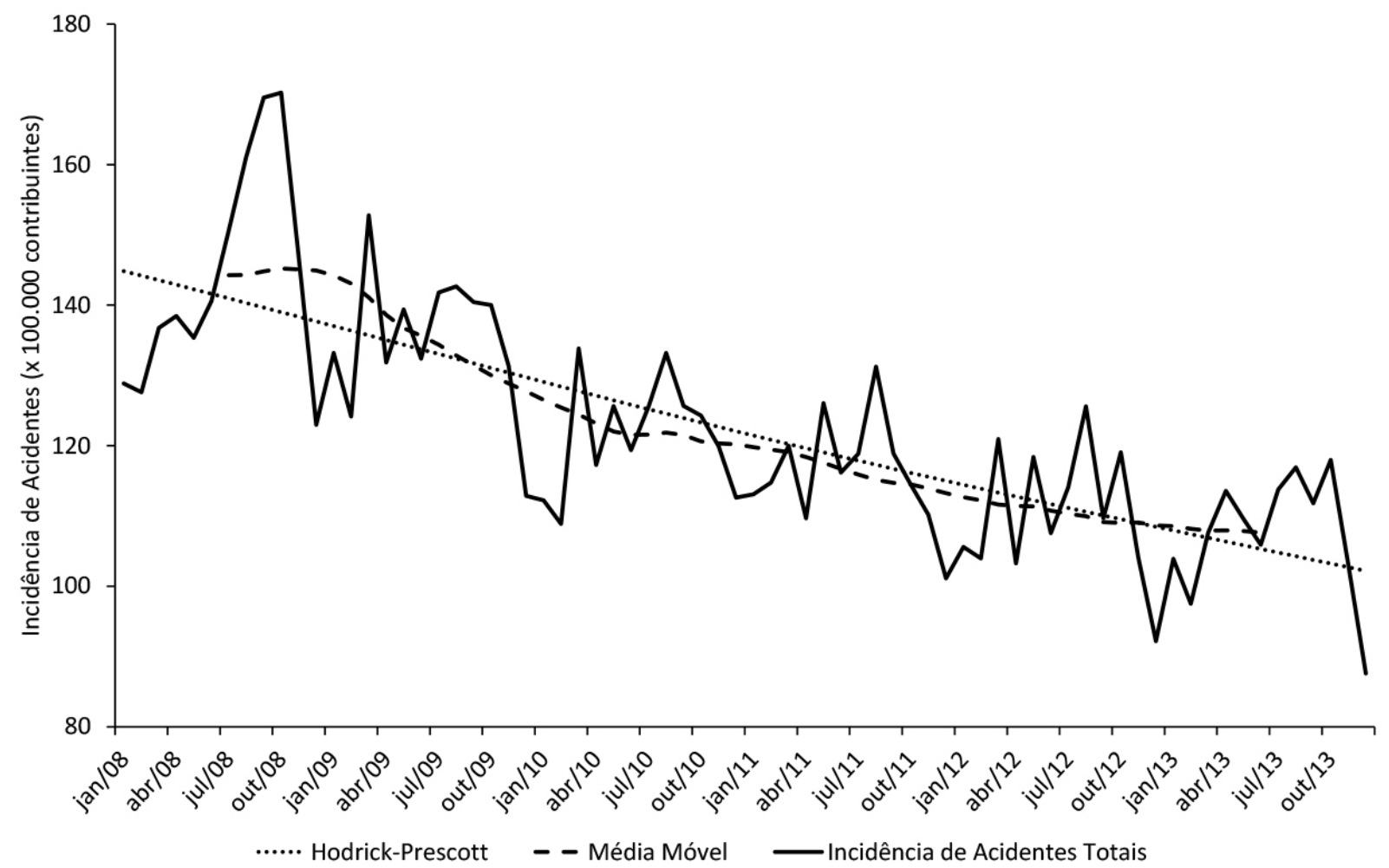

Figura 4 Nível e tendência da incidência de acidentes totais no Brasil de janeiro de 2008 a dezembro de 2013 
Tabela 1 Estatísticas descritivas para a incidência de acidentes típicos, de trajeto, de doenças ocupacionais e de acidentes totais no Brasil de janeiro de 2008 a dezembro de 2013 ( $\times 100.000$ contribuintes).

\begin{tabular}{|c|c|c|c|c|}
\hline Estatística & $\begin{array}{c}\text { Acidentes } \\
\text { típicos }\end{array}$ & $\begin{array}{c}\text { Acidentes de } \\
\text { trajeto }\end{array}$ & $\begin{array}{c}\text { Doenças do } \\
\text { trabalho }\end{array}$ & $\begin{array}{c}\text { Acidentes } \\
\text { totais }\end{array}$ \\
\hline Mínimo & 53,98 & 13,66 & 1,60 & 87,57 \\
\hline $1^{\circ}$ quartil & 66,15 & 15,20 & 2,50 & 111,40 \\
\hline Mediana & 70,39 & 16,68 & 2,84 & 119,20 \\
\hline Média & 72,21 & 16,46 & 3,00 & 122,50 \\
\hline $3^{\circ}$ quartil & 78,28 & 17,48 & 3,49 & 132,60 \\
\hline Máximo & 96,70 & 19,46 & 3,49 & 170,20 \\
\hline Teste de Dickey-Fuller Aumentado (p-valor) & 0,01 & 0,01 & 0,06 & 0,02 \\
\hline
\end{tabular}

Fonte: AEPS - Dataprev

Pela análise das Figuras, pode-se inferir que houve queda na incidência de acidentes típicos, de doenças do trabalho e de acidentes totais no período estudado, restando quantificar e analisar qual o comportamento dessa tendência quando expurgados os efeitos das variações cíclicas. No caso dos acidentes de trajeto, indica um comportamento aparentemente estável.

Quanto às estatísticas descritivas, percebe-se que, desconsideradas as referentes aos acidentes totais, a incidência mais elevada foi a de acidentes típicos, relativamente mais frequentes que doenças do trabalho. Entretanto, essa afirmação pode ser relativizada por algumas particularidades nos números de doenças do trabalho em relação aos demais acidentes, como por exemplo:

1) A quantidade de contribuintes empregados inscritos na Previdência Social pode não ser o melhor indicador da população exposta, dada a natureza específica de algumas doenças do trabalho;

2) As doenças do trabalho tendem a ser mais subnotificadas do que outros acidentes em geral, devido às dificuldades que apresentam para o estabelecimento do nexo causal.

Destacam-se também os acidentes de trajeto que, apesar de menos numerosos que os acidentes típicos, representam grande parcela do total de acidentes.

Em relação à análise de estacionaridade, com base nos resultados apresentados na Tabela 1, pode-se inferir que a incidência de acidentes típicos e de trajeto são estacionárias a 5\%, e, no caso de doenças do trabalho, a $10 \%$.

\section{Extração da tendência via decomposição}

As tendências das séries de acidente de trabalho extraídas por meio dos métodos de MM e pelo filtro
HP estão apresentadas nas Figuras 1 a 4 . O valor de estimado pelo pacote $m$ Filter para os quatro casos foi de 129.600 .

\section{Discussão}

Por meio de análise visual, foi possível identificar algumas diferenças nos resultados obtidos pela aplicação das metodologias HP e de MM. Tanto nos casos de acidentes típicos, de doenças ocupacionais quanto de acidentes totais, ambas as metodologias indicaram tendência claramente decrescente na incidência dessa ocorrência no Brasil. Entretanto, destaca-se a capacidade do modelo HP de retirar uma tendência que permita visualizar o comportamento uniforme de queda no valor da variável em todo o período de forma mais "suavizada". Por outro lado, os resultados obtidos pelo método de MM também indicam queda persistente no número dessas ocorrências. Contudo, a tendência apresenta flutuações ao redor da estimativa realizada por meio do filtro HP, assemelhando-se muito ao valor real da série.

O resultado obtido para a incidência de acidentes de trajeto permite inferir uma conclusão não corroborada pela maioria dos estudos especializados da área, com indicativo de relativa estabilidade durante todo o período. A análise obtida via método de $\mathrm{MM}$ não permite uma visualização clara da tendência no período, indicando queda no valor médio de incidência de acidentes de trajeto até 2012, seguindo-se posterior elevação acentuada. No caso do filtro HP, a tendência também diminuiu até 2012, porém com baixa amplitude de variação, o que gerou redução final de $2 \%$ do valor inicialmente observado. Portanto, apesar de ambas as tendências indicarem comportamento semelhante em termos de direção do movimento da variável ao longo do período avaliado, a magnitude das flutuações foi muito diferente, com comportamento muito mais suavizado da tendência extraída 
via HP, o que implica estabilidade em relação à tendência extraída pelo método de MM. Essa diferença fica ainda mais evidente se comparados os períodos após 2012, dado que a tendência extraída pelo filtro HP mostra estabilidade muito marcante, enquanto o modelo de MM indica elevação muito intensa.

O uso do filtro HP e do método MM permite avaliar melhor o comportamento da tendência da série relativa a acidentes de trabalho, dado que esses instrumentos estatísticos permitem "separar" o movimento da tendência de uma série dos demais componentes cíclicos que a compõe. Alguns estudos indicam a superioridade dos métodos de decomposição que levam em conta uma tendência estocástica na decomposição, já que essa especificação é mais flexível e mais compatível com fenômenos reais, tal como descrito em Sampaio ${ }^{23}$. A maior acurácia do filtro HP, quando comparado aos métodos normalmente utilizados, permite aos gestores de políticas públicas uma avaliação mais adequada da dinâmica da incidência de acidentes de trabalho no Brasil.

Há várias metodologias de extração de tendência baseadas em regressão linear, não linear e autorregressão. Entretanto, elas demandam a intervenção do pesquisador para obter o modelo estimado mais consistente, por meio de ajustes ou inclusões/exclusões de variáveis explicativas. Um estudo dessas metodologias pode ser encontrado em Enders ${ }^{20}$.

Os resultados obtidos aqui permitem inferir que o filtro HP apresenta algumas características que o diferencia dos métodos tradicionais de extração da componente tendência. Entre essas diferenças, destacam-se duas:

- A tendência não estocástica inferida via filtro HP garante abordagem não linear para a tendência de uma série temporal. Isso permite uma visualização mais acurada de processos nos quais o pesquisador tenha forte convicção de que não apresentam comportamento linear.

- A forma de extração da tendência é feita de forma mais "suavizada", podendo ser visualizada como uma "média das médias" estimada para a tendência. Essa característica permite visualizar a tendência no que concerne a seu comportamento em longo prazo.

Os resultados obtidos pelo filtro HP e pelo método MM aplicados às séries de incidência de acidentes típicos, de doenças do trabalho e de acidentes totais são consistentes com a maior parte dos achados da literatura, tal como o descrito por Wünsch Filho ${ }^{6}$, dado que ambos indicaram tendência uniforme de queda no valor da variável. Entretanto, o método MM não indicou queda uniforme no valor médio da variável no período, sendo que sua tendência apresentou flutuação semelhante à série original, sendo muito afetada por valores de curto prazo. Pode-se inferir que, devido ao número de observações, o método MM aplicado à série apresentou capacidade reduzida de extrair o comportamento em longo prazo da variável, aproximando o valor da tendência estimada ao valor da variável "incidência de acidentes de trabalho", tal como previsto por Guimarães et al. ${ }^{7}$.

Essa queda na incidência de acidentes típicos, totais e de doenças ocupacionais pode ser explicada por várias razões, sendo muitas delas descritas por Santana ${ }^{4}$. Pode-se citar como exemplo a mecanização de muitos processos produtivos nos últimos anos, o que retirou a mão de obra de algumas atividades perigosas. A subnotificação de acidentes de trabalho também é uma importante explicação para esse fenômeno, fato discutido por Cordeiro et al. ${ }^{24}$. Além disso, houve crescimento expressivo nos índices de formalidade no mercado de trabalho nos últimos anos, o que implica menor taxa de acidentes devido ao aumento no denominador (número de pessoas que se tornaram contribuintes da Previdência Social) no cálculo dessa estatística.

Cabe destacar que usar o número de vínculos de trabalho para se ter um valor aproximado da população exposta a acidentes de trabalho apresenta algumas limitações, como é o caso, por exemplo, dos trabalhadores do setor de saúde, onde é muito comum o trabalhador ter mais de um vínculo empregatício.

A importância do uso do filtro HP na análise dessas estatísticas foi propiciar visualização mais suave da dinâmica da variável, o que pode ser interpretado como seu comportamento em longo prazo, ao contrário do método MM que aproximou o valor da tendência do valor real da variável, com destaque para flutuações de curto prazo. Esses resultados estão em consonância com relatório do Banco Central do Brasil $(\text { Bacen })^{25}$, que avalia como uma das grandes vantagens do filtro HP, com relação a outras metodologias de extração de tendência, sua ampla capacidade de obter esse componente de forma mais suave do que as demais, o que permite inferir o comportamento da tendência em um intervalo de tempo mais longo.

Esses resultados são corroborados pela aplicação do filtro HP na série relativa à incidência de acidentes de trajeto. A tendência extraída via MM indica elevação acentuada na estatística a partir de 2012, o que vai ao encontro das conclusões de Almeida ${ }^{8}$. Entretanto, o resultado não foi observado com essa intensidade com a filtragem HP, que extraiu uma tendência aparentemente estável durante todo o período, especialmente após 2012, cuja amplitude de variação no nível de incidência foi da ordem de $5 \times 10^{-7}$. Tal como ocorrido na avaliação dos acidentes típicos e doenças do trabalho, a filtragem via HP 
mostra tendência de comportamento médio ao longo do tempo.

Além disso, ressalta-se a importância deste trabalho como estudo metodológico da aplicação do filtro HP nas áreas de epidemiologia e da saúde. O filtro HP já foi utilizado como instrumento em algumas pesquisas da área. Por exemplo, Ionides, Wang e Granados ${ }^{26}$ avaliaram o impacto do ciclo econômico na mortalidade, utilizando o filtro HP como forma de retirar a tendência não linear das variáveis em análise para evitar inconsistências e o enviesamento dos resultados. Doblhammer, Berg e Fritze $^{27}$ avaliaram como o ciclo econômico do momento do nascimento afetava as habilidades cognitivas desses indivíduos após os 60 anos de idade utilizando a mensuração de "ciclo" baseado no componente aleatório do ciclo de HP. Myrskylä28 estudou como condições iniciais de vida, até os 5 anos de idade, afetam a tendência de mortalidade dos indivíduos até os 30 anos de idade, sendo o filtro HP utilizado para analisar essa tendência. No entanto, cabe destacar que nenhum desses estudos teve como objetivo avaliar o comportamento do filtro HP em variáveis epidemiológicas relacionadas a acidentes de trabalho, quando comparado a outras formas de análise.

Os resultados aqui obtidos têm algumas limitações importantes. Como descrito no próprio relatório do Bacen $^{25}$, o filtro HP e o método MM são métodos mecânicos que não levam em conta outras variáveis que explicam o fenômeno em estudo, ou seja, variáveis que afetam a quantidade de acidentes de trabalho no Brasil. Entre outros autores, Putnam e Galea ${ }^{29}$ foram pioneiros na discussão dos eventos macroeconômicos e sociais que afetam variáveis importantes em estudos epidemiológicos, como os de acidentes de trabalho. Seguiram-se poucos trabalhos que retomaram os objetivos propostos por esses autores, como Wünsch Filho ${ }^{6}$, que avaliou o impacto do crescimento econômico sobre a quantidade de acidentes de trabalho, e Giovanis e Drogalas ${ }^{30}$, que fizeram uma análise da correlação de várias variáveis macroeconômicas com condições de saúde e segurança no trabalho. Os resultados obtidos por esses trabalhos devem ser incorporados por outros que visem avaliar a dinâmica da variável relativa à incidência de acidentes de trabalho, de forma a tornar a análise mais robusta e consistente. Contudo, cabe destacar que a inclusão de variáveis explicativas que levem em conta o cenário econômico e social acabaria por inviabilizar a geração de procedimentos automáticos de extração de tendência de uma série.

Outra limitação diz respeito à baixa capacidade do modelo em prever e antecipar movimentos futuros da variável em estudo. Isso decorre de outras limitações já levantadas e destacadas ao longo do texto:
- Banco de dados de acidentes de trabalho com muitas limitações, como a não inclusão de trabalhadores informais, empregados por conta própria, militares, servidores públicos estatutários, entre outras exclusões já citadas.

- Método mecânico e automático, baseado somente na evolução histórica da própria variável, sem consideração de cenários econômicos e sociais envolvidos.

Assim, cabe destacar que os resultados obtidos servem ao propósito de avaliar somente a série em estudo, ou seja, indicadores de acidentes relativos ao banco de dados da Previdência Social. Com efeito, trata-se de um banco de dados importante para o governo federal, que é utilizado como base para estudos, análises e proposições de políticas públicas aplicadas à Saúde e Segurança no Trabalho (SST). Dessa forma, o desenvolvimento de instrumentos automáticos que permitam extrair a tendência de séries estatísticas pode ser muito útil para a gestão pública.

\section{Considerações finais}

A escolha do método de extração de tendência tende a ser muito influenciada pela quantidade de observações disponíveis, bem como pelo interesse do pesquisador. O filtro HP mostra-se mais adequado em avaliar tendência em pequenas amostras, propiciando comportamento mais "suave", ao contrário do método MM, que tende a ser muito afetado por flutuações de curto prazo.

Isto é potencialmente importante para o gestor de políticas públicas, pois o uso do filtro HP permite inferir de maneira mais consistente a tendência de médio e longo prazo de uma variável relativa à incidência de acidentes de trabalho. Com base na tendência observada, o gestor poderia antecipar políticas públicas com foco nas regiões, setores e/ou tipo de acidentes com maior indicativo de crescimento no futuro. Se a análise estivesse fundamentada em um método tradicional, que não possui as características do filtro HP, como o método de MM, o gestor poderia ter suas decisões influenciadas por flutuações de curto prazo que o método extrai como tendência. Assim, o método proposto favorece uma melhor análise preditiva e a tomada oportuna de decisão, além de poder fortalecer a capacidade de atuação eficaz, efetiva e eficiente do Estado nas questões envolvidas. Esse potencial está implícito e intrinsicamente relacionado aos objetivos últimos de sistemas de informação estatais descritos em Salim, Chagas e Servo ${ }^{31}$.

O presente trabalho não esgotou as possibilidades de pesquisas para avaliar a aplicação de 
instrumentos econométricos específicos não usualmente usados no estudo de problemas da área de SST. Estudos futuros podem ser ampliados com testes de outras metodologias computacionalmente automatizáveis de extração de tendência de uma

\section{Referências}

1. Brasil. Lei 8.213, de 24 de julho de 1991. Dispõe sobre os Planos de Benefícios da Previdência Social e dá outras providências [internet]. Diário Oficial da União 25 jul 1991 [citado em 2016 fev 17]. Disponível em: http://www.planalto. gov.br/ccivil_03/leis/L8213cons.htm

2. Almeida PCA, Barbosa-Branco A. Acidentes de trabalho no Brasil: prevalência, duração e despesa previdenciária dos auxílios-doença. Rev Bras Saúde Ocup. 2011;36(124):195-207.

3. Brasil. Ministério da Previdência Social. Anuário Estatístico da Previdência Social 2013 [internet]. Brasília, DF; 2013 [citado em 2016 fev 17]. Disponível em: http:/www.previdencia.gov.br/ dados-abertos/aeps-2013-anuario-estatistico-daprevidencia-social-2013.

4. Santana V, Nobre L, Waldvogel BC. Acidentes de trabalho no Brasil entre 1994 e 2004: uma revisão. Ciênc Saúde Coletiva. 2005;10:841-55.

5. Pereira ES. Ministério da Previdência Social: informação para a saúde e segurança do trabalhador no Brasil. In: Chagas AMR, Salim CA, Servo LMS, editores. Saúde e segurança no trabalho no Brasil: aspectos institucionais, sistemas de informação e indicadores. São Paulo: Fundacentro; 2012. p. 201-31.

6. Wünsch Filho V. Reestruturação produtiva e acidentes de trabalho no Brasil: estrutura e tendências. Cad Saúde Pública. 1999;15(1):41-51.

7. Guimarães RM, Muzi CD, Ayres ARG, Ribeiro MS, Chagas C, Oliveira, JSC. Aplicação de três técnicas para avaliação de tendência de mortalidade por câncer do colo do útero em série temporal no Brasil, 1980-2009. Rev Bras Cancerol. 2012;58(3):359-67.

8. Almeida FS, Morrone LC, Ribeiro KB. Tendências na incidência e mortalidade por acidentes de trabalho no Brasil. Cad Saúde Pública. 2014;30(9):1957-64.

9. Kydland FE, Prescott EC. Time to build and aggregate fluctuations. Econometrica. 1982;50(6):1345-70.

10. Siqueira AHB, Molion LCB. Utilização do filtro de Hodrick Prescott na análise de dados climáticos. Rev Bras Climatol. 2012;8(11):25-41.

11. Organização Internacional do Trabalho. La prevención de las enfermidades profesionales. $1^{\mathrm{a}}$ ed. Genebra: OIT; 2013. série temporal, como o método de Beveridge-Nelson e o filtro de Baxter King, por exemplo. Além disso, poderiam ser realizados testes com outras variáveis importantes na área de SST, como os valores gastos com benefícios acidentários.
12. Brasil. Ministério da Previdência Social. Anuário Estatístico de Acidentes de Trabalho [internet]. Brasília, DF; 2013 [citado em 2016 ago 21]. Disponível em: http://dados.gov.br/dataset/anuarioestatistico-de-acidentes-de-trabalho

13. Ferreira LE, Ascari RA, Farias AAP. Perfil dos acidentes de trabalho registrados pelo Instituto Nacional do Seguro Social de 2007 a 2009. Rev Cient CENSUPEG. 2013;2:162-73.

14. Instituto Brasileiro de Geografia e Estatística (IBGE). Pesquisa Mensal de Emprego [internet]. Brasília, DF: IBGE; 2016 [citado em 2016 jun. 5]. Disponível em: http:/www.ibge.gov.br/home/ estatistica/indicadores/trabalhoerendimento/pme_ nova/default.shtm

15. Mendes R, Campos ACC. Saúde e segurança no trabalho informal: desafios e oportunidades para a indústria brasileira. Rev Bras Med Trab. 2004;2(3):209-23.

16. Gomes CM, Thedim-Costa SMF. Precarização do trabalho e desproteção social: desafios para a saúde coletiva. Ciên Saúde Coletiva. 1999;4(2):411-21.

17. Neto EN, Bittencourt WS, Nasrala MLS, Sousa FP, Roder IB. A influência do Nexo Técnico Epidemiológico Previdenciário sobre as notificações de LER/DORT no INSS. UNOPAR Cient Ciên Biol Saúde. 2014;16(3):209-12.

18. Brasil. Lei 11.430, de 26 de dezembro de 2006 [internet]. Diário Oficial da União 27 dez 2006 [citado em 2016 jun 10]. Disponível em: http:// www.planalto.gov.br/ccivil_03/_Ato20042006/2006/Lei/L11430.htm

19. Todeschini R, Lino D, Melo LEA. O Ministério da Previdência Social e a institucionalidade no campo da saúde do trabalhador. In: Chagas AMR, Salim, CA, Servo LMS, editores. Saúde e segurança no trabalho no Brasil: aspectos institucionais, sistemas de informação e indicadores. São Paulo: Fundacentro; 2012. p. 77-88.

20. Enders W. Applied econometric time series. 4th ed. Nova Jersey: Wiley; 2014.

21. R Development Core Team. R: A language and environment for statistical computing [internet]. Vienna: R Foundation for Statistical Computing; 2011 [citado em 2016 jun 10]. Disponível em: http://www.R-project.org/

22. Chagas AMR, Servo LMS, Salim CA. Indicadores da saúde e segurança no trabalho: fontes de dados e aplicações. In: Chagas AMR, Salim, CA, Servo LMS, editores. Saúde e segurança no trabalho 
no Brasil: aspectos institucionais, sistemas de informação e indicadores. São Paulo: Fundacentro; 2012. p. 289-329.

23. Sampaio AV. Análises de ciclos econômicos no Brasil (1980-2009). Rev Econ Tecnol. 2009;5(18):39-46.

24. Cordeiro R, Prestes SCC, Clemente APG, Diniz CS, Sakate M, Donalisio MR. Subnotificação de acidentes do trabalho não fatais em Botucatu, SP, 2002. Rev Saúde Pública. 2005;39(2):254-60.

25. Brasil. Banco Central do Brasil. Relatório de inflação: setembro de 1999 [internet]. 2011 [citado em 2016 jun 10]. Disponível em: http://www.bcb. gov.br/htms/relinf/direita.asp?idioma $=$ P\&ano $=199$ 9\&acaoAno $=$ ABRIR\&mes $=09 \&$ acaoMes $=$ ABRIR

26. Ionides EL, Wang Z, Granados JAT. Macroeconomic effects on mortality revealed by panel analysis with nonlinear trends. Ann Appl Stat. 2013;7(3):1362-85.

27. Doblhammer G, van den Berg GJ, Fritze T. Economic conditions at the time of birth and cognitive abilities late in life: evidence from ten European countries. PloS One. 2013;8(9):e74915.

28. Myrskylä M. The effects of shocks in early life mortality on later life expectancy and mortality compression: A cohort analysis. Demographic Res. 2010;22(12):289-320.

29. Putnam S, Galea S. Epidemiology and the macrosocial determinants of health. J Public Health Policy. 2008;29(3):275-89.

30. Giovanis NE, Drogalas GA. Labour relations and working conditions. Determinants using macroeconomic indicators: a quantitative research. Hum Resour Manage. 2014;2(1):1-8.

31. Salim CA, Chagas AMR, Servo LMS. Sistemas de informação e estatísticas sobre saúde e segurança no trabalho: questões, perspectivas e proposição à integração. In: Chagas AMR, Salim CA, Servo LMS, editores. Saúde e segurança no trabalho no Brasil: aspectos institucionais, sistemas de informação e indicadores. São Paulo: Fundacentro; 2012. p. 331-61. 